TAIWANESE JOURNAL OF MATHEMATICS

Vol. 14, No. 1, pp. 107-122, February 2010

This paper is available online at http://www.tjm.nsysu.edu.tw/

\title{
SOME WEIGHTED OPIAL-TYPE INEQUALITIES ON TIME SCALES
}

\author{
H. M. Srivastava, Kuei-Lin Tseng, Shio-Jenn Tseng and Jen-Chieh Lo
}

\begin{abstract}
Motivated essentially by several recent investigations which claimed to have generalized, improved and extended such classical inequalities as the well-known Opial's inequality, here we establish some general weighted Opialtype inequalities on time scales. We also provide counterexamples, corrections and modifications of the aforementioned recent claims by Wong et al.
\end{abstract}

[Taiwanese J. Math., 12 (2008), 463-471].

\section{INTRODUCTION}

Almost five decades ago, Opial [12] established an integral inequality which we recall here as Theorem A below (see also a sequel by Olech [11] for a simpler proof under weaker conditions as well as for the explicit extremal function).

Theorem A. Let $f \in C^{1}[0, a](a>0)$ with

$$
f(0)=f(a)=0 \quad \text { and } \quad f(x)>0 \quad(0<x<a) .
$$

Then

$$
\int_{0}^{a}\left|f(x) f^{\prime}(x)\right| d x \leqq \frac{a}{4} \int_{0}^{a}\left|f^{\prime}(x)\right|^{2} d x
$$

where the constant factor $\frac{a}{4}$ is the best possible. Equality holds true in (1) if and only if

$$
f(x)= \begin{cases}c x & \left(0 \leqq x \leqq \frac{a}{2}\right) \\ c(a-x) & \left(\frac{a}{2} \leqq x \leqq a\right),\end{cases}
$$

Received December 13, 2008, accepted December 15, 2008.

Communicated by J. C. Yao.

2000 Mathematics Subject Classification: Primary 26D15, Secondary 26A15, 26-99.

Key words and phrases: Opial's inequality, Extremal functions, Hölder's inequality, Time scales, $r d$-Continuous functions, Delta differentiability, Absolute continuity, Extremal functions.

*Corresponding author 
where $c$ is a positive constant.

The inequality (1) is well-known in the literature as Opial's inequality. For some recent results which generalize, improve and extend this classical inequality (1), see (for example) $[1-5,8-10,13,14,17]$ and [18] (see also the edited volume [15]). In particular, Yang [18] established the following Opial-type inequalities.

Theorem B. Let the function $f(x)$ be absolutely continuous on $[0, a](a>0)$ with $f(0)=0$. Then, for

$$
\ell, m \in \mathbb{R}:=(-\infty, \infty),
$$

each of the following inequalities holds true under the additional conditions stated with it:

$$
\begin{gathered}
\int_{0}^{a}|f(x)|^{\ell} \cdot\left|f^{\prime}(x)\right|^{m} d x \leqq\left(\frac{m}{\ell+m}\right) a^{\ell} \int_{0}^{a}\left|f^{\prime}(x)\right|^{\ell+m} d x \\
(f(0)=0 ; \quad \ell \geqq 0 ; m \geqq 1)
\end{gathered}
$$

$$
\begin{gathered}
\int_{0}^{a}|f(x)|^{\ell} \cdot\left|f^{\prime}(x)\right|^{m} d x \leqq\left(\frac{m}{\ell+m}\right) a^{\ell} \int_{0}^{a}\left|f^{\prime}(x)\right|^{\ell+m} d x \\
(f(a)=0 ; \quad \ell \geqq 0 ; m \geqq 1)
\end{gathered}
$$

and

$$
\begin{gathered}
\int_{0}^{a}|f(x)|^{\ell} \cdot\left|f^{\prime}(x)\right|^{m} d x \leqq\left(\frac{m}{\ell+m}\right)\left(\frac{a}{2}\right)^{\ell} \int_{0}^{a}\left|f^{\prime}(x)\right|^{\ell+m} d x \\
(f(0)=f(a)=0 ; \quad \ell \geqq 0 ; m \geqq 1) .
\end{gathered}
$$

By applying the Time Scales Theory and the concept of Delta Differentiability (see Section 2 below for the details of the definitions and notations used here), Agarwal et al. [2] extended the Opial's inequality (1) to the following form.

Theorem C. Let the function $f(t)$ given by

$$
f:[0, a] \cap \mathbb{T} \rightarrow \mathbb{R} \quad(a>0)
$$

be delta differentiable on $[0, a]$. Then

$$
\int_{0}^{a}\left|\left[f(t)+f^{\sigma}(t)\right] f^{\Delta}(t)\right| \Delta t \leqq a \int_{0}^{a}\left|f^{\Delta}(t)\right|^{2} \Delta t .
$$


Equality holds true in (5) when $f(t)=c t$ for a constant $c$.

In a more recent investigation, Wong et al. [17] presented several generalizations and variants of the inequality (5) for certain general cases involving time scales as asserted by Theorems D, E, F and G below.

Theorem D. Let the function $f(t)$ given by

$$
f:[a, b] \cap \mathbb{T} \rightarrow \mathbb{R} \quad(b>a \geqq 0)
$$

be delta differentiable on $[a, b] \cap \mathbb{T}$. Suppose also that

$$
p \geqq 0, \quad q \geqq 1 \quad \text { and } \quad h(t) \in C_{\mathrm{rd}}([a, b],[1, \infty)),
$$

where $C_{\mathrm{rd}}([a, b],[1, \infty))$ denotes the set of rd-continuous functions defined by

$$
C_{\mathrm{rd}}([a, b],[1, \infty)):=\{\mathfrak{f} \mid \mathfrak{f}:[a, b] \rightarrow[1, \infty) \text { and }
$$

$\mathfrak{f}(t)$ is an rd-continuous function $\}$.

Then

$$
\begin{aligned}
& \int_{a}^{b} h(t)|f(t)|^{p} \cdot\left|f^{\Delta}(t)\right|^{q} \Delta t \\
\leqq & \left(\frac{q}{p+q}\right)(b-a)^{p} \int_{a}^{b} h(t)\left|f^{\Delta}(t)\right|^{p+q} \Delta t .
\end{aligned}
$$

Theorem E. Let the function $f(t)$ given by

$$
f:[a, b] \cap \mathbb{T} \rightarrow \mathbb{R} \quad(b>a \geqq 0)
$$

be delta differentiable $n$ times $(n \in \mathbb{N})$ on $[a, b] \cap \mathbb{T}$, where

$$
\mathbb{N}:=\{1,2,3, \cdots\}
$$

Suppose also that

$$
p \geqq 0, \quad q \geqq 1 \quad \text { and } \quad h(t) \in C_{\mathrm{rd}}([a, b],[1, \infty)) .
$$

If

$$
f(a)=f^{\Delta}(a)=\cdots=f^{\Delta^{n-1}}(a)=0 \quad(n \in \mathbb{N}),
$$

then

$$
\begin{aligned}
& \int_{a}^{b} h(t)|f(t)|^{p} \cdot\left|f^{\Delta^{n}}(t)\right|^{q} \Delta t \\
\leqq & \left(\frac{q}{p+q}\right)\left[(b-a)^{p}\right]^{n} \int_{a}^{b} h(t)\left|f^{\Delta^{n}}(t)\right|^{p+q} \Delta t .
\end{aligned}
$$


Theorem F. Let each of the functions $f(t)$ and $g(t)$ given by

$$
f, g:[a, b] \cap \mathbb{T} \rightarrow \mathbb{R} \quad(b>a \geqq 0)
$$

be delta differentiable $n$ times $(n \in \mathbb{N})$ on $[a, b] \cap \mathbb{T}$. Suppose also that

$$
p \geqq 0, \quad q \geqq 1 \quad \text { and } \quad h(t) \in C_{\mathrm{rd}}([a, b],[1, \infty)) .
$$

If the function $f(t)$ satisfies the conditions in (8) and the function $g(t)$ satisfies the following conditions:

$$
g(a)=g^{\Delta}(a)=\cdots=g^{\Delta^{n-1}}(a)=0 \quad(n \in \mathbb{N}),
$$

then

$$
\begin{aligned}
& \int_{a}^{b} h(t)\left\{|f(t)|^{p} \cdot\left|g^{\Delta^{n}}(t)\right|^{q}+|g(t)|^{p} \cdot\left|f^{\Delta^{n}}(t)\right|^{q}\right\} \Delta t \\
\leqq & \left(\frac{2 q}{p+q}\right)\left[(b-a)^{p}\right]^{n} \\
& \cdot \int_{a}^{b} h(t)\left\{\left|f^{\Delta^{n}}(t)\right|^{p+q}+\left|g^{\Delta^{n}}(t)\right|^{p+q}\right\} \Delta t .
\end{aligned}
$$

Theorem G. Let each of the functions $f(t)$ and $g(t)$ given by

$$
f, g:[a, b] \cap \mathbb{T} \rightarrow \mathbb{R} \quad(b>a \geqq 0)
$$

be delta differentiable $n$ times $(n \in \mathbb{N})$ on $[a, b] \cap \mathbb{T}$. Suppose also that

$$
\frac{a+b}{2} \in[a, b], \quad p \geqq 0, \quad q \geqq 1 \quad \text { and } \quad h(t) \in C_{\mathrm{rd}}([a, b],[1, \infty)) .
$$

If the function $f(t)$ satisfies the conditions in (8) as well as the following conditions:

$$
f(b)=f^{\Delta}(b)=\cdots=f^{\Delta^{n-1}}(b)=0 \quad(n \in \mathbb{N}),
$$

and if the function $g(t)$ satisfies the conditions in (10) as well as the following conditions:

$$
g(b)=g^{\Delta}(b)=\cdots=g^{\Delta^{n-1}}(b)=0 \quad(n \in \mathbb{N}),
$$

then

$$
\begin{aligned}
& \int_{a}^{b} h(t)\left\{|f(t)|^{p} \cdot\left|g^{\Delta^{n}}(t)\right|^{q}+|g(t)|^{p} \cdot\left|f^{\Delta^{n}}(t)\right|^{q}\right\} \Delta t \\
\leqq & \left(\frac{2 q}{p+q}\right)\left[\left(\frac{b-a}{2}\right)^{p}\right]^{n} \\
& \cdot \int_{a}^{b} h(t)\left\{\left|f^{\Delta^{n}}(t)\right|^{p+q}+\left|g^{\Delta^{n}}(t)\right|^{p+q}\right\} \Delta t .
\end{aligned}
$$


The object of this paper is to show that Theorems D, E, F and G above are not valid as asserted by Wong et al. [17, Section 2] by presenting some counterexamples for them. We also prove several general weighted Opial-type inequalities on time scales. Some of our results are intended to provide corrections and modifications of the aforementioned assertions by Wong et al. [17, Section 2].

\section{A Set of Counterexamples}

Since the derivations of Theorems E, F and G by Wong et al. [17, Section 2] are all based essentially upon Theorem $\mathrm{D}$, it would suffice our purpose to give a counterexample for Theorem D only. Indeed, if (in Theorem D) we set

$$
\mathbb{T}=\mathbb{R}, p=q=1, a=0, b=r \quad(r>0), f(t)=t \quad \text { and } \quad h(t)=t+1,
$$

then the left-hand side of the inequality (7) becomes

$$
\int_{0}^{r}(t+1) t d t=\frac{r^{3}}{3}+\frac{r^{2}}{2} \quad(r>0)
$$

whereas the right-hand side of the inequality (7) assumes the following form:

$$
\frac{1}{2} r \int_{0}^{r}(t+1) d t=\frac{r^{3}}{4}+\frac{r^{2}}{2} \quad(r>0) .
$$

Upon substituting from (15) and (16) into (7), we readily arrive at a contradiction in the inequality (7) for all $r>0$. Thus, obviously, Theorem $\mathrm{D}$ (and hence also Theorems E, F and G) do not hold true as asserted by Wong et al. [17, Section 2].

We remark in passing that a special case of Theorem $\mathrm{E}$ when $n=1$, a special case of Theorem $\mathrm{F}$ when $f(t)=g(t)$, and a special case of Theorem $\mathrm{G}$ when

$$
n=1 \quad \text { and } \quad f(t)=g(t)
$$

all correspond to the erroneous inequality (7) asserted by Theorem D.

\section{Definitions, Notations and Preliminaries in Time Scales Theory and Delta DifFERENTIABILITY}

In this section, we present some definitions, notations and preliminaries concerning the Time Scales Theory and the concept of Delta Differentiability. These concepts, together with the notion of rd-continuity, were used in Section 1 above. 
Definition 1. A time scale $\mathbb{T}$ is a nonempty closed subset of $\mathbb{R}$, the two most popular examples being

$$
\mathbb{T}=\mathbb{R} \quad \text { and } \quad \mathbb{T}=\mathbb{Z}:=\{0, \pm 1, \pm 2, \cdots\} .
$$

The forward jump operator $\sigma: \mathbb{T} \rightarrow \mathbb{T}$ and the backward jump operator $\rho: \mathbb{T} \rightarrow \mathbb{T}$ are defined by

$$
\sigma(t):=\inf \{\tau \mid \tau \in \mathbb{T} \text { and } \tau>t\} \quad(t \in \mathbb{T} ; t<\sup \{\mathbb{T}\})
$$

and

$$
\rho(t):=\sup \{\tau \mid \tau \in \mathbb{T} \text { and } s<t\} \quad(t \in \mathbb{T} ; t>\inf \{\mathbb{T}\}),
$$

respectively, each of which is being supplemented by

$$
\inf \{\varnothing\}=\sup \{\mathbb{T}\} \quad \text { and } \quad \sup \{\varnothing\}=\inf \{\mathbb{T}\} .
$$

Furthermore, a point $t \in \mathbb{T}$ is called right-scattered, right-dense, left-scattered or left-dense if

$$
\sigma(t)>t, \quad \sigma(t)=t, \quad \rho(t)<t \quad \text { or } \quad \rho(t)=t,
$$

respectively.

Definition 2. Let the time scale $\mathbb{T}$ have a right-scattered minimum $\mathfrak{m}$. Then we define the set $\mathbb{T}^{\kappa}$ by

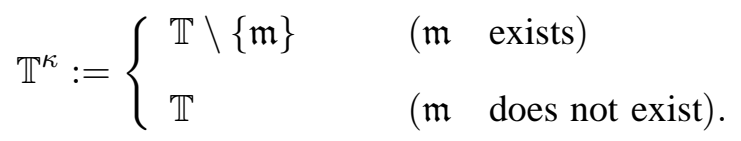

On the other hand, if the time scale $\mathbb{T}$ has a left-scattered maximum $\mathfrak{M}$, then we define the set $\mathbb{T}^{\kappa}$ by

$$
\mathbb{T}^{\kappa}:=\left\{\begin{array}{lll}
\mathbb{T} \backslash\{\mathfrak{M}\} & (\mathfrak{M} & \text { exists }) \\
\mathbb{T} & (\mathfrak{M} \quad \text { does not exist }) .
\end{array}\right.
$$

Moreover, the forward graininess $\mu: \mathbb{T} \rightarrow[0, \infty)$ is defined by

$$
\mu(t):=\sigma(t)-t \quad(t \in \mathbb{T})= \begin{cases}0 & (\mathbb{T}=\mathbb{R}) \\ 1 & (\mathbb{T}=\mathbb{Z})\end{cases}
$$

and the backward graininess $\nu: \mathbb{T} \rightarrow[0, \infty)$ is defined by

$$
\nu(t):=t-\rho(t) \quad(t \in \mathbb{T})= \begin{cases}0 & (\mathbb{T}=\mathbb{R}) \\ 1 & (\mathbb{T}=\mathbb{Z})\end{cases}
$$


Definition 3. A mapping $f: \mathbb{T} \rightarrow \mathbb{R}$ is said to be regressive if

$$
1+\mu(t) \mathfrak{f}(t) \neq 0 \quad(t \in \mathbb{T}) .
$$

Furthermore, if $\mathfrak{f}: \mathbb{T} \rightarrow \mathbb{R}$, then the mapping $\mathfrak{f}^{\sigma}: \mathbb{T} \rightarrow \mathbb{R}$ is defined by

$$
\mathfrak{f}^{\sigma}(t):=\mathfrak{f}(\sigma(t)) \quad(t \in \mathbb{T}),
$$

where $\sigma(t)$ is given in Definition 1 above.

Definition 4. A mapping $\mathfrak{f}: \mathbb{T} \rightarrow \mathbb{R}$ is said to be rd-continuous if it satisfies each of the following conditions:

(i) $f$ is continuous at every right-dense point or maximal point of $\mathbb{T}$;

(ii) The left-sided limit:

$$
\lim _{\tau \rightarrow t-} \mathfrak{f}(\tau)=\mathfrak{f}(t-)
$$

exists at every left-dense point of $\mathbb{T}$.

Just as in Equation (6) above, the space of all rd-continuous functions from $\mathbb{T} \rightarrow \mathbb{R}$ is denoted as follows:

$$
C_{\mathrm{rd}}(\mathbb{T}, \mathbb{R}):=\{\mathfrak{f} \mid \mathfrak{f}: \mathbb{T} \rightarrow \mathbb{R} \text { and } \mathfrak{f}(t) \text { is an rd-continuous function }\} .
$$

Definition 5. Assume that $\mathfrak{f}: \mathbb{T} \rightarrow \mathbb{R}$. Then we define $\mathfrak{f}^{\Delta}(t)$ to be the number (if it exists) with the property that, for any given $\epsilon>0$, there is a neighborhood $\mathcal{N}$ of $t$ such that

$$
\left|\mathfrak{f}(\sigma(t))-\mathfrak{f}(\tau)-\mathfrak{f}^{\Delta}(t)[\sigma(t)-\tau]\right| \leqq \epsilon|\sigma(t)-\tau| \quad(\tau \in \mathcal{N}) .
$$

In this case, we say that $\mathfrak{f}^{\Delta}(t)$ is the delta derivative of $\mathfrak{f}(t)$ at the point $t \in \mathbb{T}^{\kappa}$. If $\mathfrak{f}$ is delta differentiable for every $t \in \mathbb{T}^{\kappa}$, then $\mathfrak{f}$ is delta differentiable on $\mathbb{T}$ and $\mathfrak{f}^{\Delta}(t)$ is a new function defined on $\mathbb{T}^{\kappa}$.

If $\mathfrak{f}$ is delta differentiable at $t \in \mathbb{T}^{\kappa}$, then it is easily seen that

$$
\mathfrak{f}^{\Delta}(t)= \begin{cases}\lim _{\tau \rightarrow t(\tau \in \mathbb{T})} \frac{\mathfrak{f}(t)-\mathfrak{f}(\tau)}{t-\tau} & (\mu(t)=0) \\ \frac{\mathfrak{f}(\sigma(t))-\mathfrak{f}(t)}{\mu(t)} & (\mu(t)>0) .\end{cases}
$$

Several useful delta derivative formulas are recorded here under Lemma 1 below.

Lemma 1. The above-defined delta derivatives satisfy each of the following properties:

$$
f(\sigma(t))=f(t)+\mu(t) f^{\Delta}(t)
$$




$$
(f(t) g(t))^{\Delta}=f^{\Delta}(t) g(t)+f(\sigma(t)) g^{\Delta}(t)
$$

and

$$
\left(\frac{f(t)}{g(t)}\right)^{\Delta}=\frac{f^{\Delta}(t) g(t)-f(t) g^{\Delta}(t)}{g(t) g(\sigma(t))}
$$

Lemma 2 below is an easy consequence of the property (20) asserted by Lemma 1.

Lemma 2. If $f: \mathbb{T} \rightarrow \mathbb{R}$ is rd-continuous at $t \in \mathbb{T}$ and $t$ is right-scattered, then

$$
f^{\Delta}(t)=\frac{f(\sigma(t))-f(t)}{\sigma(t)-t}
$$

Definition 6. A function $\mathfrak{F}: \mathbb{T} \rightarrow \mathbb{R}$ is said to be an antiderivative of $\mathfrak{f}: \mathbb{T} \rightarrow \mathbb{R}$ if

$$
\mathfrak{F}^{\Delta}(t)=\mathfrak{f}(t) \quad\left(t \in \mathbb{T}^{\kappa}\right) .
$$

In this case, we define the integral of $f$ by

$$
\int_{s}^{t} \mathfrak{f}(\tau) \Delta \tau=\mathfrak{F}(t)-\mathfrak{F}(s) \quad(s, t \in \mathbb{T})
$$

and we say that $\mathfrak{f}$ is integrable on $\mathbb{T}$.

The results asserted by Lemma 3 below are rather immediate consequences of (21) and (24).

Lemma 3. Each of the following integral formulas holds true:

$$
\left(\int_{a}^{t} f(\tau) \Delta \tau\right)^{\Delta}=f(t)
$$

and

$$
\int_{a}^{t} f(\tau) g^{\Delta}(\tau) \Delta \tau=\left.f(\tau) g(\tau)\right|_{\tau=a} ^{t}-\int_{a}^{t} f^{\Delta}(\tau) g(\sigma(\tau)) \Delta \tau
$$

for any constant $a \in \mathbb{T}$.

\section{The Main Opial-type Inequalities on Time Scales}

We begin this section by proving our first Opial-type inequality on time scales. 
Theorem 1. Let $f:[a, b] \cap \mathbb{T} \rightarrow \mathbb{R}$ be defined as in Theorem $\mathrm{D}$. Also let

$$
p \geqq 0 \quad \text { and } \quad q \geqq 1,
$$

and suppose that $h(t)$ is a right-continuous, positive and non-increasing function on $[a, \tau] \cap \mathbb{T}$. Then the Opial-type inequality (7) holds true.

Proof. Suppose that the function $g(t)$ is given by

$$
g(t)=\int_{a}^{t}[h(s)]^{\frac{q}{p+q}}\left|f^{\Delta}(s)\right|^{q} \Delta s \quad(t \in[a, \tau] \cap \mathbb{T}),
$$

so that

$$
g(a)=0 \quad \text { and } \quad g^{\Delta}(t)=[h(t)]^{\frac{q}{p+q}}\left|f^{\Delta}(t)\right|^{q} .
$$

In the case when $q>1$, by using Hölder's inequality with indices

$$
q \quad \text { and } \quad \frac{q}{q-1}
$$

we have

$$
\begin{aligned}
|f(t)| & \leqq \int_{a}^{t}\left|f^{\Delta}(s)\right| \Delta s \\
& =\int_{a}^{t}[h(s)]^{-\frac{1}{p+q}}[h(s)]^{\frac{1}{p+q}}\left|f^{\Delta}(s)\right| \Delta s \\
& \leqq\left[\int_{a}^{t}\left([h(s)]^{-\frac{1}{p+q}}\right)^{\frac{q}{q-1}} \Delta s\right]^{\frac{q-1}{q}}\left[\int_{a}^{t}\left([h(s)]^{\frac{1}{p+q}}\left|f^{\Delta}(s)\right|\right)^{q} \Delta s\right]^{\frac{1}{q}} \\
& \left.\leqq\left[(h(t)]^{-\frac{1}{p+q}}\right)^{\frac{q}{q-1}}\left(\int_{a}^{t} 1 \cdot \Delta s\right)\right]^{\frac{q-1}{q}}[g(t)]^{\frac{1}{q}} \\
& =[h(t)]^{-\frac{1}{p+q}}(t-a)^{\frac{q-1}{q}}[g(t)]^{\frac{1}{q}},
\end{aligned}
$$

which readily yields

$$
[h(t)]^{\frac{p}{p+q}}|f(t)|^{p} \leqq(t-a)^{\frac{p(q-1)}{q}}[g(t)]^{\frac{p}{q}} .
$$

In the case when $q=1$, we find that

$$
\begin{aligned}
|f(t)| & \leqq \int_{a}^{t}\left|f^{\Delta}(s)\right| \Delta s \\
& =\int_{a}^{t}[h(s)]^{-\frac{1}{p+1}}[h(s)]^{\frac{1}{p+1}}\left|f^{\Delta}(s)\right| \Delta s \\
& \leqq[h(t)]^{-\frac{1}{p+1}} \int_{a}^{t}[h(s)]^{\frac{1}{p+1}}\left|f^{\Delta}(s)\right| \Delta s \\
& =[h(t)]^{-\frac{1}{p+1}} g(t),
\end{aligned}
$$


which shows that the inequality (28) holds true also when $q=1$. Thus, by means of (27) and (28), we observe that

$$
\begin{aligned}
& \int_{a}^{\tau} h(t)|f(t)|^{p}\left|f^{\Delta}(t)\right|^{q} \Delta t \\
= & \int_{a}^{\tau}[h(t)]^{\frac{p}{p+q}}|f(t)|^{p}[h(t)]^{\frac{q}{p+q}}\left|f^{\Delta}(t)\right|^{q} \Delta t \\
\leqq & \int_{t=a}^{\tau}(t-a)^{\frac{p(q-1)}{q}}[g(t)]^{\frac{p}{q}} g^{\Delta}(t) \Delta t \\
\leqq & (\tau-a)^{\frac{p(q-1)}{q}} \int_{a}^{\tau}[g(t)]^{\frac{p}{q}} \Delta g(t) \\
\leqq & (\tau-a)^{\frac{p(q-1)}{q}}\left(\frac{q}{p+q}\right)[g(\tau)]^{\frac{p+q}{q}},
\end{aligned}
$$

since (by definition) $g(a)=0$ as given by (27).

On the other hand, from Hölder's inequality with indices

$$
\frac{p+q}{p} \quad \text { and } \quad \frac{p+q}{q},
$$

we obtain

$$
\begin{aligned}
g(\tau) & =\int_{a}^{\tau}[h(t)]^{\frac{q}{p+q}}\left|f^{\Delta}(t)\right|^{q} \Delta t \\
& \leqq\left(\int_{a}^{\tau} 1^{\frac{p+q}{p}} \cdot \Delta t\right)^{\frac{p}{p+q}}\left[\int_{a}^{\tau}\left([h(t)]^{\frac{q}{p+q}}\left|f^{\Delta}(t)\right|^{q}\right)^{\frac{p+q}{q}} \Delta t\right]^{\frac{q}{p+q}} \\
& \leqq(\tau-a)^{\frac{p}{p+q}}\left(\int_{a}^{\tau} h(t)\left|f^{\Delta}(t)\right|^{p+q} \Delta t\right)^{\frac{q}{p+q}} .
\end{aligned}
$$

Therefore, we finally have

$$
\begin{aligned}
& \int_{a}^{\tau} h(t)|f(t)|^{p}\left|f^{\Delta}(t)\right|^{q} \Delta t \\
\leqq & \left(\frac{q}{p+q}\right)(\tau-a)^{p} \int_{a}^{\tau} h(t)\left|f^{\Delta}(t)\right|^{p+q} \Delta t,
\end{aligned}
$$

which evidently completes the proof of Theorem 1 .

Theorem 2. Let the function $f:[a, b] \cap \mathbb{T} \rightarrow \mathbb{R}$ be delta differentiable with $f(b)=0$. Also let

$$
p \geqq 0 \quad \text { and } \quad q \geqq 1,
$$


and suppose that $h(t)$ is a right-continuous, positive and non-increasing function on $[\tau, b] \cap \mathbb{T}$. Then

$$
\begin{aligned}
& \int_{\tau}^{b} h(t)|f(t)|^{p}\left|f^{\Delta}(t)\right|^{q} \Delta t \\
\leqq & \left(\frac{q}{p+q}\right)(b-\tau)^{p} \int_{\tau}^{b} h(t)\left|f^{\Delta}(t)\right|^{p+q} \Delta t .
\end{aligned}
$$

Proof. We consider a function $g(t)$ given by

$$
g(t)=\int_{t}^{b}[h(s)]^{\frac{q}{p+q}}\left|f^{\Delta}(s)\right|^{q} \Delta s \quad(t \in[\tau, b] \cap \mathbb{T})
$$

so that

$$
g(b)=0 \quad \text { and } \quad g^{\Delta}(t)=-[h(t)]^{\frac{q}{p+q}}\left|f^{\Delta}(t)\right|^{q} .
$$

In the case when $q>1$, by using Hölder's inequality with indices

$$
q \quad \text { and } \quad \frac{q}{q-1}
$$

we get

$$
\begin{aligned}
|f(t)| & \leqq \int_{t}^{b}\left|f^{\Delta}(s)\right| \Delta s \\
& =\int_{t}^{b}[h(s)]^{-\frac{1}{p+q}}[h(s)]^{\frac{1}{p+q}}\left|f^{\Delta}(s)\right| \Delta s \\
& \leqq\left[\int_{t}^{b}\left([h(s)]^{-\frac{1}{p+q}}\right)^{\frac{q}{q-1}} \Delta s\right]^{\frac{q-1}{q}}\left[\int_{t}^{b}\left([h(s)]^{\frac{1}{p+q}}\left|f^{\Delta}(s)\right|\right)^{q} \Delta s\right]^{\frac{1}{q}} \\
& \left.\leqq\left[(h(t)]^{-\frac{1}{p+q}}\right)^{\frac{q}{q-1}}\left(\int_{t}^{b} 1 \cdot \Delta s\right)\right]^{\frac{q-1}{q}}[g(t)]^{\frac{1}{q}} \\
& =[h(t)]^{-\frac{1}{p+q}}(b-t)^{\frac{q-1}{q}}[g(t)]^{\frac{1}{q}},
\end{aligned}
$$

which implies the following inequality:

$$
[h(t)]^{\frac{p}{p+q}}|f(t)|^{p} \leqq(b-t)^{\frac{p(q-1)}{q}}[g(t)]^{\frac{p}{q}} .
$$


In the case when $q=1$, we have

$$
\begin{aligned}
|f(t)| & \leqq \int_{t}^{b}\left|f^{\Delta}(s)\right| \Delta s \\
& =\int_{t}^{b}[h(s)]^{-\frac{1}{p+1}}[h(s)]^{\frac{1}{p+1}}\left|f^{\Delta}(s)\right| \Delta s \\
& =[h(t)]^{-\frac{1}{p+1}} \int_{t}^{b}[h(s)]^{\frac{1}{p+1}}\left|f^{\Delta}(s)\right| \Delta s \\
& =[h(t)]^{-\frac{1}{p+1}} g(t),
\end{aligned}
$$

which shows that the inequality (31) holds true also when $q=1$. Thus, by (30) and (31), we find that

$$
\begin{aligned}
\int_{\tau}^{b} h(t)|f(t)|^{p}\left|f^{\Delta}(t)\right|^{q} \Delta t & =\int_{\tau}^{b}[h(t)]^{\frac{p}{p+q}}|f(t)|^{p}[h(t)]^{\frac{q}{p+q}}\left|f^{\Delta}(t)\right|^{q} \Delta t \\
& \leqq \int_{\tau}^{b}(b-t)^{\frac{p(q-1)}{q}}[g(t)]^{\frac{p}{q}}\left[-g^{\Delta}(t)\right] \Delta t \\
& \leqq(b-\tau)^{\frac{p(q-1)}{q}} \int_{t=\tau}^{b}\left(-[g(t)]^{\frac{p}{q}}\right) \Delta g(t) \\
& \leqq\left.(b-\tau)^{\frac{p(q-1)}{q}}\left(\frac{q}{p+q}\right)\left(-[g(t)]^{\frac{p+q}{q}}\right)\right|_{t=\tau} ^{b} \\
& \leqq(b-\tau)^{\frac{p(q-1)}{q}}\left(\frac{q}{p+q}\right)[g(\tau)]^{\frac{p+q}{q}},
\end{aligned}
$$

since (by definition) $g(b)=0$ as given by (30).

On the other hand, in view of Hölder's inequality with indices

$$
\frac{p+q}{p} \quad \text { and } \quad \frac{p+q}{q},
$$

we have

$$
\begin{aligned}
g(\tau) & =\int_{\tau}^{b}[h(t)]^{\frac{q}{p+q}}\left|f^{\Delta}(t)\right|^{q} \Delta t \\
& \leqq\left(\int_{\tau}^{b} 1^{\frac{p+q}{p}} \cdot \Delta t\right)^{\frac{p}{p+q}}\left[\int_{\tau}^{b}\left([h(t)]^{\frac{q}{p+q}}\left|f^{\Delta}(t)\right|^{q}\right)^{\frac{p+q}{q}} \Delta t\right]^{\frac{q}{p+q}} \\
& \leqq(b-\tau)^{\frac{p}{p+q}}\left(\int_{\tau}^{b} h(t)\left|f^{\Delta}(t)\right|^{p+q} \Delta t\right)^{\frac{q}{p+q}} .
\end{aligned}
$$


Therefore, we finally obtain

$$
\begin{aligned}
& \int_{\tau}^{b} h(t)|f(t)|^{p}\left|f^{\Delta}(t)\right|^{q} \Delta t \\
\leqq & \left(\frac{q}{p+q}\right)(b-\tau)^{p} \int_{\tau}^{b} h(t)\left|f^{\Delta}(t)\right|^{p+q} \Delta t,
\end{aligned}
$$

which is precisely the inequality (29) asserted by Theorem 2 .

Theorem 3. Let the function $f:[a, b] \cap \mathbb{T} \rightarrow \mathbb{R}$ be delta differentiable with

$$
f(a)=f(b)=0 .
$$

Also let

$$
p \geqq 0, \quad q \geqq 1 \quad \text { and } \quad \tau \in[a, b] \cap \mathbb{T} .
$$

Furthermore, suppose that the function $h(t)$ is positive and non-increasing on $[a, \tau] \cap \mathbb{T}$ and non-increasing on $[\tau, b] \cap \mathbb{T}$. Then

$$
\begin{aligned}
& \int_{a}^{b} h(t)|f(t)|^{p}\left|f^{\Delta}(t)\right|^{q} \Delta t \\
\leqq & \left(\frac{q}{p+q}\right)(\tau-a)^{p} \int_{a}^{\tau} h(t)\left|f^{\Delta}(t)\right|^{p+q} \Delta t \\
& +\left(\frac{q}{p+q}\right)(b-\tau)^{p} \int_{\tau}^{b} h(t)\left|f^{\Delta}(t)\right|^{p+q} \Delta t .
\end{aligned}
$$

Proof. By Theorem 1 and 2, we get

$$
\begin{aligned}
\int_{a}^{b} h(t)|f(t)|^{p}\left|f^{\Delta}(t)\right|^{q} \Delta t= & \int_{a}^{\tau} h(t)|f(t)|^{p}\left|f^{\Delta}(t)\right|^{q} \Delta t \\
& +\int_{\tau}^{b} h(t)|f(t)|^{p}\left|f^{\Delta}(t)\right|^{q} \Delta t \\
\leqq & \left(\frac{q}{p+q}\right)(\tau-a)^{p} \int_{a}^{\tau} h(t)\left|f^{\Delta}(t)\right|^{p+q} \Delta t \\
& +\left(\frac{q}{p+q}\right)(b-\tau)^{p} \int_{\tau}^{b} h(t)\left|f^{\Delta}(t)\right|^{p+q} \Delta t
\end{aligned}
$$

which obviously completes the proof of Theorem 3 .

By setting

$$
\tau=\frac{a+b}{2}
$$


in Theorem 3, we arrive at an interesting special case as given below.

Corollary. The following weighted Opial-type inequality holds true on time scales:

$$
\begin{aligned}
& \int_{a}^{b} h(t)|f(t)|^{p}\left|f^{\Delta}(t)\right|^{q} \Delta t \\
\leqq & \left(\frac{q}{p+q}\right)\left(\frac{b-a}{2}\right)^{p} \int_{a}^{b} h(t)\left|f^{\Delta}(t)\right|^{p+q} \Delta t .
\end{aligned}
$$

Theorems 4, 5 and 6 below can be proven by employing the same methods as those used in the proofs of Theorems E, F and G, respectively (see, for details, [17]).

Theorem 4. Let the function $f$ be defined as in Theorem E. Also let $p, q$ and the function $h(t)$ be defined as in Theorem 1. Then the inequality (9) holds true.

Theorem 5. Let the functions $f$ and $g$ be defined as in Theorem F. Also let $p$, $q$ and the function $h(t)$ be defined as in Theorem 1. Then the inequality (11) holds true.

Theorem 6. Let the functions $f$ and $g$ be defined as in Theorem G. Also let $p$, $q$ and the function $h(t)$ be defined as in Theorem 1. Then the inequality (14) holds true.

\section{ACKNOWLEDGMENTS}

The present investigation was supported, in part, by the Natural Sciences and Engineering Research Council of Canada under Grant OGP0007353.

\section{REFERENCES}

1. R. P. Agarwal, Sharp Opial-type inequalities involving $r$-derivatives and their applications, Tohoku Math. J. (Ser. 2), 47 (1995), 567-593.

2. R. Agarwal, M. Bohner and A. Peterson, Inequalities on time scales: A survey, Math. Inequal. Appl., 4 (2001), 535-557.

3. R. P. Agarwal and P. Y. H. Pang, Remarks on the generalizations of Opial's inequality, J. Math. Anal. Appl., 190 (1995), 559-577.

4. R. P. Agarwal and P. Y. H. Pang, Opial Inequalities with Applications in Differential and Difference Equations, Series on Mathematics and Its Applications, Vol. 320, Kluwer Academic Publishers, Dordrecht, Boston and London, 1995. 
5. P. R. Beesack, On an integral inequality of Z. Opial, Trans. Amer. Math. Soc., 104 (1962), 470-475.

6. M. Bohner and A. Peterson, Dynamic Equations on Time Scales: An Introduction with Applications, Birkhäuser, Boston, 2001.

7. M. Bohner and A. Peterson (Editors), Advances in Dynamic Equations on Time Scales, Birkhäuser, Boston, 2003.

8. K. M. Das, An inequality similar to Opial's inequality, Proc. Amer. Math. Soc., 20 (1969), 258-261.

9. X. G. He, A short proof of a generalization on Opial's inequality, J. Math. Anal. Appl., 182 (1994), 299-300.

10. C. L. Mallows, An even simpler proof of Opial's inequality, Proc. Amer. Math. Soc., 16 (1965), 173-173.

11. C. Olech, A simple proof of a certain result of Z. Opial, Ann. Polon. Math., 8 (1960), 61-63.

12. Z. Opial, Sur une inégalité, Ann. Polon. Math., 8 (1960), 29-32.

13. B. G. Pachpatte, On Opial-type integral inequalities, J. Math. Anal. Appl., 120 (1986), 547-556.

14. J. E. Pečaric, An integral inequality, in Analysis, Geometry and Groups: A Riemann Legacy Volume (H. M. Srivastava and Th. M. Rassias, Editors), Part II, pp. 471-478, Hadronic Press, Palm Harbor, Florida, 1993.

15. Th. M. Rassias and H. M. Srivastava (Editors), Analytic and Geometric Inequalities and Applications, Series on Mathematics and Its Applications, Vol. 478, Kluwer Academic Publishers, Dordrecht, Boston and London, 1999.

16. D. Willett, The existence-uniqueness theorem for an $n$th order linear ordinary differential equation, Amer. Math. Monthly, 75 (1968), 174-178.

17. F.-H. Wong, W.-C. Lian, S.-L. Yu and C.-C. Yeh, Some generalizations of Opial's inequalities on time scales, Taiwanese J. Math., 12 (2008), 463-471.

18. G.-S. Yang, On a certain result of Z. Opial, Proc. Japan Acad., 42 (1966), 78-83.

H. M. Srivastava

Department of Mathematics and Statistics,

University of Victoria,

Victoria, British Columbia V8W 3R4,

Canada

E-mail: harimsri@math.uvic.ca 


\section{Kuei-Lin Tseng}

Department of Mathematics,

Aletheia University,

Tamsui 25103, Taiwan

E-mail: kltseng@email.au.edu.tw

Shio-Jenn Tseng and Jen-Chieh Lo

Department of Mathematics,

Tamkang University,

Tamsui 25137, Taiwan

E-mail: topology@mail.tku.edu.tw m8813026@yahoo.com.tw 